\section{Additions to the known larval host plants of Indian Lepidoptera}

\section{Peter Smetacek ${ }^{1}$ \& Rajni Smetacek ${ }^{2}$}

1,2 The Butterfly Research Centre, The Retreat, Jones Estate, P.O.Bhimtal, District Nainital, Uttarakhand 263136 India Email: ${ }^{1}$ petersmetacek@rediffmail.com (corresponding author); 2rajnismetacek@rediffmail.com

The larval host plants of some families of Indian Lepidoptera such as the Papilionidae, Saturniidae and Sphingidae are relatively well known (Bell \& Scott 1937; Robinson et al. 2001). Information on most of the other families is quite incomplete.

The present list consists largely of species bred by the authors at Butterfly Research Centre, Bhimtal, Nainital District, Uttarakhand, India in the Kumaon Himalaya west of Nepal $\left(29^{\circ} 20^{\prime} 41^{\prime \prime} \mathrm{N} \& 79^{\circ} 30^{\prime} 17^{\prime \prime} \mathrm{E}\right)$. Most of the species recorded were obtained as wild

Date of publication (online): 26 December 2011

Date of publication (print): 26 December 2011

ISSN $0974-7907$ (online) | 0974-7893 (print)

Editor: George Mathew

Manuscript details:

Ms \# 02745

Received 03 April 2011

Final received 15 November 2011

Finally accepted 22 November 2011

Citation: Smetacek, P. \& R. Smetacek (2011). Additions to the known larval host plants of Indian Lepidoptera. Journal of Threatened Taxa 3(12): 2272-2276.

Copyright: @ Peter Smetacek \& Rajni Smetacek 2011. Creative Commons Attribution 3.0 Unported License. JoTT allows unrestricted use of this article in any medium for non-profit purposes, reproduction and distribution by providing adequate credit to the authors and the source of publication.

Acknowledgements: We are indebted to the following persons and institutions, without whose help this work would have been impossible: the Head and staff of the Dept. of Forest Entomology, Forest Research Institute, Dehra Dun, India for permission to work on the collection in their care and to publish records; the Chief Conservator of Forests, Jammu and Kashmir for kind permission to one of us (PS) to work on hawkmoths around Leh, Ladakh and the Wildlife Warden and personnel of the Jammu and Kashmir Forest Department for their generous help there; Professor Emeritus Y.P.S. Pangtey of the Botany Department, D.S.B. College, Nainital, India for identifying plants and clearing several taxonomic problems of a botanic nature Dr. Wolfgang Speidel of the Museum Witt, Munich, Germany for identifying some Noctuids.; Dr. Wolfgang Nässig, Forschingsinstitut und Naturmuseum Senckenberg, Frankfurt am Main, Germany, for literature; Professor L.W.R. Kobes, Heterocera Sumatrana Society, Göttingen, Germany, for confirming the identity of Gabala roseoretis, Dr. I.J. Kitching, N.H.M., London, U.K. for identifying some species and advice on the placement and arrangement of the taxa; Manoj Chandran, I.F.S. for breeding Cleora reciprocaria, photographs of the early stages of Acytolepis puspa on Quercus and breeding notes on the Sesia sp.; and especially the Rufford Small Grant Foundation, U.K. for financing this work.

\section{OPEN ACGESS | FREE DOWNLOAD}

larvae in various instars from forests in Nainital District between $1200 \mathrm{~m}$ and $2400 \mathrm{~m}$ elevation. The places visited were mainly in the Bhimtal and Sattal valleys (1200-1500 m), the Bhowali Valley $(1600 \mathrm{~m})$, the Gagar pass $(2400 \mathrm{~m})$, Maheshkhan forest (2200m) and Rata forest (2200m) at all seasons, opportunistically over a period of 20 years but focussed on Maheshkhan, Gagar and Rata forests during 2008 to 2009. All the above mentioned locations are within a 50-km radius of Bhimtal. Since the same species was often encountered in different locations within the district, we have refrained from giving exact locations in the list. Voucher specimens of the material bred are in the authors' personal collection at the above address.

In addition, an invitation to the Forest Research Institute in Dehra Dun to the senior author resulted in the identification of a number of moths that had lain unidentified in the Indian National Forest Insect Collection since the 1930s. Several of these had been bred in different parts of India and Burma and the localities on their data labels are mentioned in this list. It should be noted that the names of several localities have changed during the intervening 80 years. The original names on the data labels have been noted so as to facilitate reference to these specimens, while the currently valid names have been mentioned in parenthesis. Similarly, names of plants on which the Lepidoptera were bred are, in many cases, not currently valid. Nevertheless, these have been mentioned as noted on the original data labels and the current botanical names included in parenthesis. In one case (Pseudomicronia coelata Moore), it was not possible to discover the author or family of the plant name mentioned on the data label, yet this name has been included in the hope that others are more fortuitous than the present authors. In another case, (Nephele hespera Fabricius) it was not possible to locate the authority for the species of plant (Carissa affinarium) mentioned on the moth's data label, so this name has been listed as such. All specimens in the Forest Research Institute have been distinguished by an asterisk in the following list.

The Sypnini (Erebidae) do not seem to have a subfamily placement yet (I.J. Kitching pers. comm.) so it has been placed as a Tribe in the Erebidae. 
In addition, there are eight records, one each by Antram (1924), Bailey (1951) and Troup (1899), two by Mackinnon \& de Nicéville (1897-1898) and three by Wynter-Blyth (1957) that appear to have been overlooked by subsequent authors, notably Robinson et al. (2001). These have been included in this list.

\section{Systematic section}

\section{Psychiidae}

*Eumeta Walker sp.: seeds of Pinus wallichiana A.B. Jackson (= Pinus excelsa Walich ex D. Don) (Pinaceae), Kangra, Himachal Pradesh.

\section{Tortricidae}

Archips machlopis Meyrick: Neolitsea umbrosa (Nees) Gamble (Lauraceae).

\section{Sesiidae}

Sesia Fabricius sp.: bark of Phyllanthus emblica L. (Euphorbiaceae).

\section{Limacodidae}

*Parasa lepida Cramer: Strychnos nux-vomica L. (Moraceae), Dehra Dun.

*Miresa albipuncta Herrich-Schäffer: "Dicopyros melanoxylum" = Diopyros exsculpta Buch.-Ham. (Ebenaceae), Hoshangabad, C.P. (=Hoshangabad, Madhya Pradesh).

* Cania himalayana Holloway: Bombax ceiba L. (Bombacaceae), Dehra Dun.

*Cheromettia apicata Moore: Terminalia belerica (Gaertn.) Roxb. (Combretaceae); Litchi chinensis Sonnerat (Sapindaceae), both Dehra Dun.

*Aphendala cana Walker: Citrus L. (Rutaceae),

Dehra Dun.

*Naros a conspersa Walker: Desmodium triquetrum

(L.) DC (Fabaceae), Coorg Titthimatti (=Titthimatti, Kodagu District, Karnataka).

*Altha subnotata Swinhoe: "Terminalia tomentosa"

= Terminalia alata Heyne ex Roth (Combretaceae);

Citrus L. (Rutaceae); Shorea robusta Gaertn.

(Dipterocarpaceae), all Dehra Dun.

*Chalcoscelides castaneipars Moore: "Cinchona"

$=$ ? Cinchona officinalis L. (Rubiaceae) Darjeeling, West Bengal.

*Scopelodes ursina Butler: "Aleurites montana"

= Vernicia montana Lour. (Euphorbiaceae) Lashio, Burma.

\section{Zygaeniidae}

*Gynautocera papilionaria GuérinMenéville:"Litsea polyantha" = Litsea monopetala (Roxb.) Pers. (Lauraceae) Dehra Dun.

Soritia leptalina Kollar: Bridelia montana (Roxb.) Willd. (Euphorbiaceae); Rubus ellipticus Smith (Rosaceae).

\section{Pyralidae}

Heterocrasa expansalis Warren: Quercus leucotrichophora A Camus (Fagaceae).

\section{Drepanidae}

Drepaninae

Callidrepana argenteola Moore: Rhus wallichi Hook. F. (Anacardiaceae).

Deroca hyalina Walker: Benthamidia capitata (Wallich) Hara (Cornaceae).

Deroca inconclusa Walker: Benthamidia capitata (Wallich) Hara (Cornaceae).

Drepana near quinaria Moore: Alnus nepalensis

D. Don (Betulaceae).

Oreta extensa Walker: Swida oblonga (Wallich) Soják (Cornaceae).

\section{Bombycidae}

Penicillifera lactea Hutton: Ficus neriifolia var. nemoralis (Wall. Ex Miq.) Corner (Moraceae).

\section{Saturniidae}

Actias selene Hübner: Symplocos chinensis (Lour.) Druce (Symplocaceae).

Samia canningii Hutton: Zanthoxylum armatum DC (Rutaceae).

\section{Sphingidae}

*Ambulyx liturata Butler: Shorea robusta Gaertn. (Dipterocarpaceae), Dehra Dun.

*Clanis phalaris Cramer: Ficus benghalensis L. (Moraceae), Salem, Tamil Nadu.

Polyptychus trilineatus Moore: Terminalia chebula Retz. (Combretaceae), Dehra Dun. (included in Robinson et al. (2001) with an interrogation mark.).

*Nephele hespera Fabricius: "Carissa affinarium"??

Psilogramma menephron Cramer:Tecomaria capensis (Lindl.) Spach. (Bignoniaceae).

Hyles gallii Rottemburg: Euphorbia stracheyi 
Boiss. (Euphorbiaceae), Leh, Ladakh, Jammu \& Kashmir.

Hyles nicaea lathyrus Walker: Euphorbia stracheyi

Boiss. (Euphorbiaceae), Leh, Ladakh, Jammu \& Kashmir.

*Macroglossum bombylans Boisduval:"Terminalia tomentosa" = Terminalia alata Heyne ex Roth (Combretaceae), Dehra Dun.

*Cephonodes hylas Linnaeus: Xeromphis spinosa (Thunb.) Keay (Rubiaceae), Paonta, Himachal Pradesh.

\section{Lasiocampidae}

Trabala vishnou Lef.: Coriaria nepalensis Wallich (Coriariaceae); Rubus ellipticus Smith (Rosaceae).

Malacosoma indica Walker: Salix denticulata Andersson (Salicaceae)

\section{Epicopeidae}

Epicopeia polydorus Westwood: Persea odoratissima (Nees) Kosterm. (Oleaceae).

\section{Uraniidae}

Uraniinae

*Pseudomicronia coelata Moore: "Congronema nepalensis"??, Dehra Dun.

\section{Epipleminae}

*Orudiza protheclaria Walker: Oroxylum indicum (Link) Vent. (Bignoniaceae), Dehra Dun.

*Dysaethria fulvilinea Hampson: Gmelina arborea Roxb. (Verbenaceae); Premna latifolia Roxb. (Verbenaceae), both Dehra Dun.

* Dysaethria near ruptaria Moore: Premna berbata Wall. Ex Schauer (Verbenaceae), Dehra Dun.

*Dirades adjutaria Walker: "Randia uliginosa" = Catunaregam uliginosa (Retz.) Sivarajan (Rubiaceae), Coorg (=Kodagu District, Karnataka); "Randia dumetorum" = Catunaregam spinosa (Thunb.) Tirven (Rubiaceae), Coorg (=Kodagu District, Karnataka); "Adina cordifolia" - Haldina cordifolia (Roxb.) Ridsdale (Rubiaceae), Wynaad (in Tamil Nadu); Cassia fistula L. (Leguminosae), Dehra Dun.

*Gathynia miraria Walker: Randia L. sp. (Rubiaceae), Titthimatti, Coorg (=Kodagu District, Karnataka).

\section{Geometridae}

\section{Ennominae: Orthostixini}

Naxa textilis form huegeli Felder: Osmanthus fragrans Lour. (Oleaceae) (vide Troup (1899)), Kausani, Uttarakhand.

\section{Ennominae}

Psyra spurcataria Walker: Rosa L. (Rosaceae).

Cleora reciprocaria Walker: Sapindus mukorossi Gaertn. (Sapidaceae).

Cleora repulsaria Walker: Bauhinia vareigata L. (Caesalpinaceae).

Ectropis crepuscularia Hübner: Glochidion heyneanum (Wight \& Arn.) Wight (Euphorbiaceae), Tagetes L. (Asteraceae).

\section{Larentiinae}

Phthonoloba decussata Moore: Bridelia montana (Roxb.) Willd. (Euphorbiaceae).

\section{Notodontidae}

*Phalera raya Moore: Cassia fistula L. (Leguminosae), Dehra Dun.

Dudusa sphingiformis Moore: Acer oblongum Wallich ex DC (Aceraceae).

Somera viridifusca Walker: Syzygium cuminii (L.) Skeels (Myrtaceae).

\section{Erebidae}

\section{Lymantriinae}

*Caviria ochripes Moore: Grewia sp. (Tiliaceae), Dehra Dun.

Artaxa guttata Walker: Citrus L. sp. (Rutaceae).

Euproctis varia Walker: Coriaria nepalensis Wallich (Coriariaceae).

Euproctis plagiata Walker: Syzygium cuminii (L.) Skeels (Myrtaceae).

"Euproctis” sp.: Carpinus viminea Lindley (Corylaceae).

Arctornis subvitrea Walker: Carpinus viminea Lindley (Corylaceae).

Ilema kosemponica Strand: Dioscorea bulbifera

L. (Dioscoreaceae); Impatiens edgeworthii Hook. F. (Balsaminaceae).

Olene inclusa Walker: Quisqualis indica L. (Combretaceae); Rosa L. (Rosaceae).

Olene magnalia Swinhoe: Begonia picta Smith (Begoniaceae). 
Rhypotoses drepanioides Kishida: Quercus floribunda Lindley ex A. Camus (Fagaceae)

\section{Arctiinae}

Phissama transiens Walker: Crassocephalum crepidioides (Benth.) S. Moore (Asteraceae).

Spilarctia casigneta Kollar: Cirsium verutum (D. Don) Sprengel (Asteraceae), Mirabilis jalapa L. (Nyctagenaceae); Dahlia Cav. (Asteraceae); Tagetes L. Asteraceae); Craniotome furcata (Link) O. Kuntze (Lamiaceae); Gynura bicolor (Roxb. Ex Willd.) DC (Asteraceae); Goldfussia dalhousiana Nees (Acanthaceae); Passiflora edulis Sims. (Passifloraceae); Girardinia diversifolia (Link) Friis (Urticaceae); Lantana camara L. (Verbenaceae).

*Mangina argus Kollar: "Crotolaria sericea" = Crotolaria spectabilis Roth (Fabaceae), Dehra Dun.

Nyctemera adversata Schaller: Debregeasa longifolia (Burm. f.) Wedd., Girardinia diversifolia (Link) Friis, Urtica dioica L. (all Urticaceae); Gynura bicolor (Roxb. Ex Willd.) DC (Asteraceae); Crassocephalum crepidioides (Benth.) S. Moore (Asteraceae).

\section{Calpinae}

Gonitis mesogona Walker: Rubus ellipticus Smith (Rosaceae).

Gonitis near mesogona Walker: Grewia optiva J.R. Drumm ex Burrtt. (Tiliaceae).

\section{Erebinae \\ Sypnini}

Sypna curvilinea Moore: Rubus ellipticus Smith (Rosaceae).

\section{Nolidae}

\section{Chloephorinae}

Gabala roseoretis Kobes : Acer oblongum Wallich ex DC (Aceraceae).

\section{Noctuidae}

Thiacidinae

Thiacidas ?indica Walker: Carpinus viminea Lindley (Corylaceae).

\section{Plusiinae}

Trichoplusia orichalcea Fabricius: Cirsium verutum (D. Don) Sprengel (Asteraceae).
Arcteini (currently unplaced to subfamily)

Arcte caerulea Guenee: Girardinia diversifolia (Link) Friis (Urticaceae)

\section{Nymphalidae \\ Satyrinae}

Elymnias malelas Hewitson: "leaves of banana" = Musa balbisiana Colla (Musaceae) (vide Antram 1924) locality ?Assam; the butterfly is common in banana groves in the Kumaon Himalaya.

\section{Acraeinae}

Acraea vesta Fabricius: Pouzolzia zeylanica (Linn.) Bennet \& Brown (Urticaceae).

\section{Charaxinae}

*Polyura athamas Drury: Helicteres isora L. (Sterculaceae), Coorg (=Kodagu District, Karnataka).

\section{Limenitidinae}

Neptis mahendra Moore: A female oviposited on several young leaves of a bush of Pyracantha crenulata (D. Don) M. Roemer (Rosaceae) in the field; larvae emerged but did not eat the leaves (Smetacek 2011)).

\section{Lycaenidae}

Talicada nyseus Guérin-Menéville: Kalanchoë spathulata DC (Crassulaceae).

Celastrina gigas Hemming: Prinsepia utilis Royle (Rosaceae) (Wynter-Blyth 91957); mihi).

Celastrina huegelii Moore: Prinsepia utilis Royle (Rosaceae) (Wynter-Blyth (1957); mihi).

Celastrina argiolus kollari Westwood: Prinsepia utilis Royle (Rosaceae) (Wynter-Blyth (1957)).

*Acytolepis puspa gisca Fruhstorfer: Shorea talura Roxb. (Dipterocarpaceae), Coorg (=Kodagu District, Karnataka); Quercus leucotrichophora A. Camus (Fagaceae), Nainital, Uttarakhand (identity of latter record confirmed on the basis of photographs taken by Manoj Chandran, Government Silviculturist).

Horaga albimacula viola Moore: Coriaria nepalensis Wallich (Coriariaceae) (vide Mackinnon \& de Nicéville (1897-1898)).

Dodona eugenes eugenes Bates: Myrsine semiserrata Wallich (Myrsinaceae).

Dodona dipoea nostia Fruhstorfer: Myrsine semiserrata Wallich (Myrsinaceae). 


\section{Papilionidae}

Troides aeacus C. \& R. Felder: Aristolochia dilatata N.E. Brown (Aristolochiaceae).

Atrophaneura aidoneus Doubleday: Aristolochia dilatata N.E. Brown (Aristolochiaceae).

Byasa dasarada ravana Moore: Aristolochia dilatata N.E. Brown (Aristolochiaceae).

Byasa polyeuctes Doubleday: Aristolochia dilatata N.E. Brown (Aristolochiaceae).

Papilio arcturus Westwood: Skimmia Thunb. (Rutaceae), Kathmandu, Nepal (vide Bailey (1951).

Papilio agestor govindra Moore: Persea duthiei (King ex Hook. f.) (Lauraceae).

Graphium (Pazala) eurous cashmirensis

Rothschild: Persea duthiei (King ex Hook. f.)

Kosterm., Neolitsea umbrosa (Nees) Gamble (both Lauraceae).

\section{Pieridae}

Aporia agathon agathon Gray: Berberis chitra

Edwards (Berberidaceae).

Aporia soracta Moore: Berberis chitra Edwards

(Berberidaceae).

Pieris canidia Sparrman: Cardamine impatiens L.

(Brassicaceae).

Gonepteryx rhamni nepalensis Doubleday:

Rhamnus triqueter (Wallich) Brandis (Rhamnaceae).

Colias erate Esper: ovipositing on Vicia sativa Linnaeus.

Colias fieldii Menétries: oviposited on Trifolium repens Linnaeus.

\section{Hesperiidae}

Bibasis oedipodea aegina P1.: Hiptage madablota Gaertn. (Malphigiaceae) (vide Mackinnon \& de Niceville (1897-1898)).

\section{REFERENCES}

Antram, C.B. (1924). Butterflies of India. Thacker, Spink \& Co., Calcutta \& Simla, 226pp.

Bailey, F.M. (1951). Notes on butterflies from Nepal. Journal of the Bombay Natural History Society 50: 64-87; 281-298.

Bell, T.R.D. \& F.B. Scott (1937). Fauna of British India including Ceylon and Burma. Moths-Vol. 5. Sphingidae. Taylor and Francis, London, 14+537pp+15pls+1map.

Mackinnon P.W. \& L. De Nicéville (1897-98). A list of the butterflies of Mussoorie in the Western Himalayas and neighbouring regions. Journal of the Bombay Natural History Society 11: 205-221; 368-389; 585-623, 6 pls.

Robinson, G.S., P.W. Ackery, I.J. Kitching, G.W. Beccaloni \& L.M. Hernandez (2001). Hostplants of The Moth and Butterfly Caterpillars of the Oriental Region. The Natural History Museum, London \& Southdene Sdn. Bht., Kuala Lumpur, 744pp.

Smetacek, P. (2011). A review of West Himalayan Neptini (Nymphalidae). Journal of the Lepidopterists' Society 65(3): 153-161.

Troup, N.F.T. (1899). A plague of web-making caterpillars on the "Silang" tree (Olea fragrans). Journal of the Bombay Natural History Society 12: 775-776.

Wynter-Blyth, M.A. (1957). The Butterflies of the Indian Region. Bombay Natural History Society, Bombay, $20+523 \mathrm{pp}+72 \mathrm{pls}$ 